\section{Transtornos mentais em uma amostra de gestantes da rede de atenção básica de saúde no Sul do Brasil}

\author{
Mental disorders in a sample of pregnant women \\ receiving primary health care in Southern Brazil
}

\author{
1 Faculdade de Medicina, \\ Universidade Federal do Rio \\ Grande do Sul, Porto Alegre, \\ Brasil. \\ 2 Instituto de Matemática, \\ Universidade Federal do Rio \\ Grande do Sul, Porto Alegre, \\ Brasil. \\ Correspondência \\ M. S. Almeida \\ Programa de Pós-graduação \\ em Epidemiologia, Faculdade \\ de Medicina, Universidade \\ Federal do Rio Grande do Sul. \\ Rua Amélia Telles 596, \\ apto. 304, Porto Alegre, RS \\ 90460-070, Brasil. \\ mscortegagn@globo.com
}

\section{Abstract}

The aim of this study was to examine the prevalence of probable psychiatric disorders diagnosed during pregnancy and related sociodemographic causative factors among 712 women between the 16 th and 36th week of pregnancy receiving prenatal care in 18 basic health units in Porto Alegre and Bento Gonçalves in southern Brazil. PRIME$M D$ was used to assess mental disorders. The prevalence of probable mental disorder occurred in $41.7 \%$ of the women. The most prevalent diagnosis was major depressive disorder (21.6\%), followed by generalized anxiety disorder (19.8\%). A multivariate analysis showed that the factors most significantly associated with a probable psychiatric disorder were: the fact that the pregnant woman did not work or study: $P R=1.25$ (95\%CI: 1.04-1.51); the fact that the pregnant woman did not live with her spouse: $P R=1.24$ (95\%CI: 1.011.52); the fact that the pregnant woman had two or more children: $P R=1.21$ (95\%CI: 1.01-1.46). A high prevalence of probable mental disorder was observed. The increased search for health care by pregnant women provides an opportunity for screening, diagnosing and treating these disorders under the primary health care system.

Mental Disorders; Pregnancy; Primary Health Care; Cross-Sectional Studies
Michele Scortegagna de Almeida 1

Maria Angélica Nunes 1

Suzi Camey ${ }^{2}$

Andrea Poyastro Pinheiro 1

Maria Inês Schmidt 1

\section{Introdução}

Atualmente, os transtornos mentais se constituem um problema de saúde pública. Estimativa da Organização Mundial da Saúde aponta que cerca de 450 milhões de pessoas sofram de algum transtorno mental, e que um em cada quatro indivíduos, será afetado por uma doença psiquiátrica em algum estágio de sua vida ${ }^{1}$. Acreditase, ainda, que esse número sofreu um aumento progressivo, principalmente nos países de média e baixa renda (LAMIC - low and middle income countries) incluindo também o Brasil, devido a uma associação significativa com o baixo nível socioeconômico 2 .

Dentro deste contexto, a literatura mostra que as mulheres apresentam maiores prevalências de transtornos mentais em relação aos homens, principalmente em transtornos de humor, de ansiedade, somatoformes 3,4,5,6,7,8, e comorbidades psiquiátricas 6 .

Historicamente a saúde mental da mulher grávida foi negligenciada, possivelmente influenciada por dois fatores. Um deles é a crença popular de a gravidez ser um período de bem-estar para as mulheres. Outro é a maior ênfase dada aos transtornos psicóticos ocorridos no pós-parto imediato que, por gerarem mais hospitalizações psiquiátricas, recebem maior atenção dos profissionais de saúde 9 . No entanto, os dados epidemiológicos apontam que as prevalências de transtornos mentais comuns são semelhan- 
tes na gravidez e no puerpério 10,11,12. Diferenças observadas apontaram maiores prevalências no período da gravidez em relação ao período pósparto 13,14,15,16,17, tornando a gestação uma fase crucial para diagnóstico e tratamento das patologias psiquiátricas.

Logo, há uma clara necessidade de um melhor conhecimento sobre a saúde mental da mulher durante a gravidez, uma vez que os transtornos mentais na gravidez constituem importantes preditores de depressão pós-parto 11,14,15,17,18, de ansiedade pós-parto 11, de desfechos obstétricos adversos 19,20,21,22,23, e que podem influenciar o desenvolvimento infantil, tendo reflexos até a adolescência 24 .

Estudos sobre a prevalência de transtornos mentais em grávidas e sua associação com fatores socioeconômicos ainda são escassos no Brasil $25,26,27$, uma vez que muitos avaliam apenas os transtornos depressivos 28,29,30.

O objetivo do presente estudo é estimar a prevalência de transtornos mentais na gestação em uma amostra de grávidas de duas cidades do sul do país, e analisar a sua distribuição segundo variáveis sociodemográficas.

\section{Metodologia}

Este estudo é parte do projeto Medida do Padrão de Consumo Alimentar, Prevalência de Transtornos Mentais e Violência em uma amostra de Gestantes (ECCAGE). Trata-se de um estudo de coorte que arrolou 780 gestantes entre a 16a e a 36a semana de gestação, atendidas em 18 unidades básicas de saúde (UBS), sendo 10 unidades localizadas na cidade de Porto Alegre e 8 em Bento Gonçalves, ambas no Rio Grande do Sul, Brasil. As gestantes foram selecionadas de forma consecutiva, na ocasião de sua consulta pré-natal, sendo a entrevista realizada em um único contato com as participantes. Os dados apresentados neste artigo foram coletados na linha de base do projeto. O cálculo de tamanho de amostra foi realizado com a ferramenta STATCALC do Epi Info (Centers for Disease Control and Prevention, Atlanta, Estados Unidos) para estimar o tamanho de amostra da linha de base do projeto. Originalmente, estimou-se uma prevalência de comportamentos alimentares inadequados de 10\%, com intervalo de 95\% de confiança (IC95\%) e erro de $2,3 \%$, resultando em 654 gestantes. Adicionaramse $20 \%$ ao se considerar possíveis perdas e recusas, resultando em um total de 785 participantes. Tal desfecho, comportamentos alimentares inadequados, era o desfecho de menor prevalência dentro do projeto original. Portanto, a estimativa de tamanho de amostra para os demais desfechos do consórcio, incluindo transtornos mentais comuns, foi considerada adequada por estimar um tamanho de amostra maior. Maiores detalhes sobre o protocolo do estudo e sua metodologia foram publicados anteriormente 31 .

\section{Instrumentos}

\section{- Questionário sobre dados sociodemográficos}

Trata-se de um questionário que contém as variáveis sociodemográficas idade, escolaridade, atividade remunerada, renda familiar, situação conjugal, número de habitantes na residência e paridade. Também foram coletadas informações referentes à saúde da gestante, seus hábitos durante a gestação e planejamento da atual gestação, bem como presença de doença crônica (diabetes, hipertensão), consumo de álcool e tabaco e índice de massa corporal (IMC).

\section{- PRIME-MD}

Para avaliar a presença de sintomas psiquiátricos, foi utilizado o instrumento para Avaliação de Transtornos Mentais para Atenção Primária (The Primary Care Evaluation of Mental Disorders; PRIME-MD) 32. Ele é um instrumento utilizado no rastreamento de transtornos mentais comuns em pacientes na rede de atenção básica e de fácil aplicabilidade. Foi traduzido e validado para o português brasileiro por Fráguas Jr. et al. 33. O PRIME-MD avalia os quatro grupos de transtornos mentais mais comuns encontrados na atenção básica: os de humor, de ansiedade, somatoformes e álcool e, também, os transtornos alimentares, utilizando critérios diagnósticos do DSM-III-R (Diagnostic and Statistical Manual of Mental Disorders) 34 . O módulo de transtorno somatoforme foi excluído em virtude da frequência elevada de queixas físicas em gestantes, o que confundiria o diagnóstico, além da necessidade de confirmação clínica. Neste estudo, o PRIMEMD foi aplicado por entrevistadoras treinadas, mas não habilitadas para a realização de avaliações clínicas do estado mental das gestantes.

O instrumento é dividido em duas partes: o Questionário do Paciente e o Guia de Avaliação Clínica. O Questionário do Paciente é constituído por 12 questões (sendo 10 de resposta dicotômica sim/não), e uma questão que mede a intensidade do sintoma e outra que avalia saúde global. As primeiras dez questões são organizadas em módulos, cobrindo os diagnósticos e, no caso de resposta afirmativa, o Guia de Avaliação Clínica é usado para obter informações adicionais para cada diagnóstico. A partir dos dados coletados, 
é possível identificar seis categorias diagnósticas: transtorno depressivo maior, transtorno depressivo maior em remissão parcial, distimia, transtorno do pânico, transtorno de ansiedade generalizada e bulimia nervosa. No presente estudo não houve a utilização do Guia de Avaliação Clínica. Na análise, foram incluídas somente as participantes que relataram a presença de todos os critérios de cada diagnóstico específico em relação ao DSM-III-R, indicando então, a presença de provável transtorno mental.

\section{Aspectos éticos}

O projeto foi aprovado pelo Comitê de Ética em Pesquisa da Universidade Federal do Rio Grande do Sul e todas as participantes assinaram consentimento informado.

\section{Análise estatística}

Foram estimadas as prevalências dos prováveis transtornos psiquiátricos. Para as variáveis contínuas, foram calculados a média e o desvio padrão. Foi avaliada a presença de associação entre provável transtorno psiquiátrico e as variáveis sociodemográficas e gravidez planejada através da regressão de Poisson com variância robusta 35,36 . No modelo multivariado foram incluídas as variáveis que apresentaram valor de $\mathrm{p}<0,2$ na regressão simples, sendo mantidas, no modelo final, somente as com significância menor que $5 \%$, controlando para possíveis fatores de confusão que foram a presença de doença crônica, consumo de álcool e tabaco na gestação e IMC, fatores esses disponíveis e relatados na literatura como associados a maior prevalência de transtornos mentais 20,26,30. Foram, então, estimadas as razões de prevalência brutas e ajustadas. A análise estatística foi realizada com o programa SPSS v16.0 (SPSS Inc., Chicago, Estados Unidos).

\section{Resultados}

Das 780 gestantes arroladas, 68 (8,7\%) foram classificadas como perdas e recusas, restando 712 para análise. A idade média foi 24,6 anos (variando de 13-42), 63,2\% $(\mathrm{n}=450)$ tinham menos de nove anos de estudo formal, $69,8 \%$ ( $\mathrm{n}=$ 497) tinham renda familiar inferior a 3 salários mínimos e 59\% (n = 420) não estudavam nem trabalhavam fora de casa. Quinhentas e sessenta e três $(79,1 \%)$ moravam com companheiro e $62,6 \%(n=446)$ das gestantes não planejaram a gravidez. Quanto ao número de pessoas na residência, 32\% ( $n=228)$ moravam com cinco ou mais pessoas e $27,7 \%(n=197)$ tinham dois ou mais filhos (Tabela 1). A maioria das gestantes $(72,1 \% ; n=513)$ foi entrevistada no segundo trimestre de gestação.

Duzentos e noventa e sete gestantes preenchiam critérios para ao menos um provável diagnóstico pelo PRIME-MD, resultando em 41,7\% da amostra. O diagnóstico mais prevalente foi o transtorno depressivo maior $(21,6 \%$; $\mathrm{n}=154)$, seguido pelo transtorno de ansiedade generalizada $(19,8 \%$; $n=141)$, transtorno do pânico $(9,3 \%$; $\mathrm{n}=66)$, transtorno depressivo maior em remissão parcial $(9 \%$; $n=64)$, distimia $(8,4 \% ; n=60)$ e bulimia nervosa $(0,6 \% ; \mathrm{n}=4)$. O número de diagnósticos também é relatado na Tabela 2. Dentre as 297 gestantes com provável diagnóstico de transtorno psiquiátrico, 42,4\% ( $\mathrm{n}=126)$ apresentavam comorbidade, ou seja, dois ou mais diagnósticos psiquiátricos concomitantes.

No modelo de regressão univariada, escolaridade, situação conjugal, ocupação e número de filhos aparecem como variáveis associadas a provável transtorno mental pelo PRIME-MD, conforme Tabela 3. Na análise multivariada, após ajuste para diabetes, hipertensão, tabagismo, provável abuso ou dependência de álcool e IMC, os fatores que mantiveram significância com a prevalência de provável transtorno mental foram: não trabalhar nem estudar $(\mathrm{RP}=1,25$; IC95\%: 1,04-1,51), não morar com o companheiro ( $\mathrm{RP}=1,24$; IC95\%: 1,01-1,52), e ter dois ou mais filhos (RP = 1,21; IC95\%: 1,01-1,46).

\section{Discussão}

Taxa elevada de transtornos mentais em mulheres não é um novo achado 6 . No presente estudo, observou-se que $41,7 \%$ das gestantes apresentaram provável transtorno psiquiátrico. $\mathrm{O}$ fato de a gestante não trabalhar nem estudar, não morar com o companheiro e já ter dois ou mais filhos esteve associado a maior risco de apresentar um provável diagnóstico de transtorno mental.

A prevalência elevada de transtornos mentais comuns em gestantes encontra respaldo na literatura, quando se refere às populações de baixo nível socioeconômico 17,37,38. Estudos indicam que há um excesso de transtornos mentais, particularmente transtornos de humor, em amostras populacionais e provenientes de atenção básica 6 .

A prevalência estimada de transtorno depressivo maior foi de $21,6 \%$, e de $9 \%$ para o transtorno depressivo maior em remissão parcial, totalizando $30,6 \%$, resultado superior aos provenientes de outros países do LAMIC, que variam de 20,5\% a $24,7 \% 15,17,22$ e das grávidas brasileiras $(12,9 \%$ a $20,8 \%$ ) 25,29,30,39. Causas biológicas (neuroen- 
Distribuição das características sociodemográficas e planejamento da gravidez em gestantes com e sem provável transtorno mental pelo PRIME-MD atendidas nas unidades básicas de saúde no Sul do Brasil, 2007.

\begin{tabular}{|c|c|c|c|c|c|c|}
\hline & \multicolumn{2}{|c|}{$\begin{array}{l}\text { Sem transtorno mental } \\
\qquad(n=415)\end{array}$} & \multicolumn{2}{|c|}{$\begin{array}{l}\text { Com transtorno mental } \\
\qquad(\mathrm{n}=297)\end{array}$} & \multicolumn{2}{|c|}{$\begin{array}{c}\text { Total } \\
(\mathrm{n}=712)\end{array}$} \\
\hline & $\mathrm{n}$ & $\%$ & $\mathbf{n}$ & $\%$ & $\mathrm{n}$ & $\%$ \\
\hline \multicolumn{7}{|l|}{ Idade (anos) } \\
\hline$\leq 19$ & 114 & 27,5 & 67 & 22,6 & 181 & 25,4 \\
\hline $20-29$ & 201 & 48,4 & 163 & 54,9 & 364 & 51,1 \\
\hline$>30$ & 100 & 24,1 & 67 & 22,6 & 167 & 23,5 \\
\hline \multicolumn{7}{|l|}{ Escolaridade (anos) } \\
\hline $0-4$ & 49 & 11,8 & 49 & 16,5 & 98 & 13,8 \\
\hline $5-8$ & 201 & 48,4 & 151 & 50,8 & 352 & 49,4 \\
\hline $9+$ & 165 & 39,8 & 97 & 32,7 & 262 & 36,8 \\
\hline \multicolumn{7}{|l|}{ Renda familiar (salários mínimos) } \\
\hline$\leq 1$ & 68 & 16,4 & 59 & 19,9 & 127 & 17,8 \\
\hline $1,01-3,00$ & 218 & 52,5 & 152 & 51,2 & 370 & 52,0 \\
\hline$\geq 3,01$ & 129 & 31,1 & 86 & 29,0 & 215 & 30,2 \\
\hline \multicolumn{7}{|l|}{ Ocupação } \\
\hline Trabalha e/ou estuda & 187 & 45,1 & 105 & 35,4 & 292 & 41,0 \\
\hline Não trabalha nem estuda & 218 & 54,9 & 192 & 64,6 & 420 & 59,0 \\
\hline \multicolumn{7}{|l|}{ Número de pessoas na casa } \\
\hline $1-2$ & 104 & 25,1 & 67 & 22,6 & 171 & 24,0 \\
\hline $3-4$ & 187 & 45,1 & 126 & 42,4 & 313 & 44,0 \\
\hline$\geq 5$ & 124 & 29,9 & 104 & 35,0 & 228 & 32,0 \\
\hline \multicolumn{7}{|l|}{ Situação conjugal } \\
\hline Mora com companheiro & 337 & 81,2 & 226 & 76,1 & 563 & 79,1 \\
\hline Não mora com companheiro & 78 & 18,8 & 71 & 23,9 & 149 & 20,9 \\
\hline \multicolumn{7}{|l|}{ Número de filhos } \\
\hline $0-1$ & 316 & 76,1 & 199 & 67,0 & 515 & 72,3 \\
\hline$\geq 2$ & 99 & 23,9 & 98 & 33,0 & 197 & 27,7 \\
\hline \multicolumn{7}{|l|}{ Gravidez planejada } \\
\hline Sim & 158 & 38,1 & 108 & 36,4 & 266 & 37,4 \\
\hline Não & 257 & 61,9 & 189 & 63,6 & 446 & 62,6 \\
\hline
\end{tabular}

dócrinas) e sociais (mãe solteira, ausência de suporte social adequado) são consideradas. Mulheres são particularmente vulneráveis para experimentar episódios depressivos durante o ciclo vital e em situações específicas (divórcio, pósparto, menopausa) ${ }^{6}$. Entretanto, os achados do presente estudo foram semelhantes aos encontrados em revisão sistemática conduzida com amostra de gestantes de baixo nível socioeconômico, que encontrou $28 \%$ e $25 \%$ de transtorno depressivo, para segundo e terceiro trimestres, respectivamente 40 . Já em revisão sistemática de estudos com gestantes oriundas de países ricos, a prevalência de transtorno depressivo foi de 8,5\%, para ambos os trimestres, através de entrevista clínica estruturada 10 . Os instrumentos utilizados para medir sintomas depressivos e as amostras selecionadas variaram muito nos diferentes estudos, o que pode explicar os resultados discrepantes, dificultando sua comparação.

A prevalência de Transtorno de Ansiedade Generalizada foi alta $(19,8 \%)$ sendo que estudos anteriores, realizados com o PRIME-MD em mulheres atendidas em ambulatórios de cuidados básicos, reportam prevalências de $9 \%$ e 12,3\% 4,6. A possível inclusão do transtorno de ajustamento com sintomas ansiosos nessa categoria diagnóstica poderia estar contribuindo para a prevalência elevada, já que a gravidez é reconhecida como um desencadeante do transtorno 41.

A presença de morbidade psiquiátrica é um importante achado no atual estudo com implicações clínicas. Dentre as 297 gestantes com provável diagnóstico de transtorno psiquiátrico, 42,4\% 
Prevalência de provável diagnóstico psiquiátrico e número de transtornos psiquiátricos pelo PRIME-MD em gestantes atendidas nas unidades básicas de saúde no Sul do Brasil, 2007.

\begin{tabular}{lcc}
\hline & $\mathbf{n}$ & $\%$ \\
\hline Transtorno depressivo maior & 154 & 21,6 \\
Transtorno depressivo maior em remissão parcial & 64 & 9,0 \\
Distimia & 60 & 8,4 \\
Transtorno do pânico & 66 & 9,3 \\
Transtorno de ansiedade generalizada & 141 & 19,8 \\
Bulimia nervosa & 4 & 0,6 \\
Número de transtornos mentais & & 58,3 \\
0 & 415 & 171 \\
2 & 74 & 24,0 \\
& 52 & 10,4 \\
\hline
\end{tabular}

( $\mathrm{n}=126)$ apresentavam comorbidade, ou seja, dois ou mais diagnósticos psiquiátricos concomitantes. Rastrear, diagnosticar e tratar uma gestante com um transtorno mental pode ser muito valioso na prática clínica, mas é importante assinalar para os profissionais de saúde envolvidos com o pré-natal das gestantes que poderá haver outro adicional transtorno mental, que também poderá ser adequadamente tratado.

No presente estudo, a situação conjugal da gestante (não morar com o companheiro) esteve associada a maior prevalência de transtornos psiquiátricos $(R P=1,24)$, resultado semelhante ao obtido em estudos brasileiros 25,29 e internacionais 11,16,42. Em recente revisão de literatura conduzida por Lancaster et al. ${ }^{43}$, o fato de não morar com o companheiro é fator de risco para depressão na gravidez, e esses autores ainda ressaltam como o fator de risco mais relevante a falta de suporte social do companheiro. É possível supor que não residir com o companheiro seja vivenciado como falta de apoio social, predispondo a transtorno mental, assim como não morar com companheiro pode ser uma consequência da presença de transtorno psiquiátrico.

Quanto à paridade, ter dois ou mais filhos, mostrou associação $(\mathrm{RP}=1,21)$ com transtornos mentais. Preocupações decorrentes das exigências sociais, emocionais e psicológicas de cuidar de um bebê e outras crianças ao mesmo tempo, com pouco suporte social no seu entorno, poderiam constituir fator de estresse importante para essas mulheres. Grant et al. 11 demonstraram que as mulheres que tinham mais de um filho apresentavam mais sintomas ansiosos e depressivos pós-parto. Outro trabalho encontrou associação entre o fato de ter três ou mais filhos e maior prevalência de transtornos mentais comuns 44 .

No presente estudo, gestantes que não trabalhavam nem estudavam tinham $25 \%(\mathrm{RP}=1,25)$ mais provável diagnóstico de transtorno psiquiátrico. Esse resultado é corroborado por estudos anteriores, que também encontraram uma associação entre ocupação e transtornos depressivos em gestantes 26,30,45.

Não houve associação entre baixa escolaridade e maior prevalência de transtornos mentais, conforme relatado na literatura 2,3,5,7,46,47. Esse fato pode ter sido decorrente da homogeneidade da amostra, uma vez que apenas 15 gestantes $(2,1 \%)$ tinham mais de 11 anos de escolaridade, correspondendo a nível educacional além do ensino médio completo. Estudo realizado no Canadá, que encontrou grande diferença de prevalência de sintomas depressivos em grávidas de baixo e alto nível socioeconômico, $47 \%$ e $20 \%$, respectivamente, utilizou como ponto de corte para baixo nível socioeconômico ter menos de 12 anos de estudo 38 .

O presente estudo apresenta algumas limitações: o próprio delineamento transversal impossibilita definir relação causal, uma vez que a exposição e o desfecho foram medidas simultaneamente. Outra limitação é que o PRIME-MD foi utilizado por entrevistadoras treinadas sem habilitação para a avaliação clínica do estado mental da gestante, o que implica frequentemente uma superestimativa de prevalência.

A importante vantagem da utilização do instrumento foi a ampliação dos possíveis diagnósticos psiquiátricos rastreados, visto que o PRIMEMD avalia outros diagnósticos psiquiátricos além 
Associação entre características sociodemográficas e planejamento da gravidez e suspeita de diagnóstico psiquiátrico pelo PRIME-MD das gestantes atendidas nas unidades básicas de saúde no Sul do Brasil, 2007.

\begin{tabular}{|c|c|c|c|c|}
\hline & \multicolumn{2}{|c|}{ RP bruta } & \multicolumn{2}{|c|}{ RP ajustada * } \\
\hline & RP (IC95\%) & Valor de $p$ & RP (IC95\%) & Valor de $p$ \\
\hline \multicolumn{5}{|l|}{ Idade (anos) } \\
\hline$\leq 19$ & $0,92(0,71-1,20)$ & 0,55 & & \\
\hline $20-29$ & $1,12(0,90-1,39)$ & 0,32 & & \\
\hline$>30$ & 1,00 & & & \\
\hline \multicolumn{5}{|l|}{ Escolaridade (anos) } \\
\hline $0-4$ & $1,35(1,05-1,74)$ & 0,02 & & \\
\hline $5-8$ & $1,16(0,95-1,41)$ & 0,15 & & \\
\hline $9+$ & 1,00 & & & \\
\hline \multicolumn{5}{|l|}{ Renda familiar (salários mínimos) } \\
\hline$\leq 1,00$ & $1,16(0,91-1,49)$ & 0,24 & & \\
\hline $1,01-3,00$ & $1,03(0,84-1,26)$ & 0,80 & & \\
\hline$\geq 3,01$ & 1,00 & & & \\
\hline \multicolumn{5}{|l|}{ Ocupação } \\
\hline Trabalha e/ou estuda & 1,00 & & 1,00 & \\
\hline Não trabalha nem estuda & $1,27(1,06-1,53)$ & 0,01 & $1,25(1,04-1,51)$ & 0,02 \\
\hline \multicolumn{5}{|l|}{ Número de pessoas na casa } \\
\hline $1-2$ & 1,00 & & & \\
\hline $3-4$ & $1,03(0,82-1,29)$ & 0,82 & & \\
\hline$\geq 5$ & $1,16(0,92-1,47)$ & 0,20 & & \\
\hline \multicolumn{5}{|l|}{ Situação conjugal } \\
\hline Mora com companheiro & 1,00 & & 1,00 & \\
\hline Não mora com companheiro & $1,19(0,98-1,44)$ & 0,09 & $1,24(1,01-1,52)$ & 0,04 \\
\hline \multicolumn{5}{|l|}{ Número de filhos } \\
\hline $0-1$ & 1,00 & & 1,00 & \\
\hline$\geq 2$ & $1,29(1,08-1,54)$ & 0,01 & $1,21(1,01-1,46)$ & 0,04 \\
\hline \multicolumn{5}{|l|}{ Gravidez planejada } \\
\hline Sim & 1,00 & & & \\
\hline Não & $1,04(0,87-1,25)$ & 0,64 & & \\
\hline
\end{tabular}

* Corrigido para fumo na gravidez, provável abuso ou dependência de álcool, índice de massa corporal, diabetes e hipertensão.

dos transtornos mentais comuns (ansiosos e depressivos).

Os resultados aqui descritos não podem ser generalizados para toda população brasileira, mas parecem representar adequadamente as gestantes que realizam seu pré-natal em serviços públicos de saúde no sul do país, uma vez que a amostra é proveniente de 18 unidades de cuidados básicos de duas cidades 48 .

O presente estudo demonstrou a magnitude do problema, uma vez que a amostra foi constituída de gestantes com uma média de 24 anos e em situação de extrema vulnerabilidade (não trabalhar nem estudar, não morar com o companheiro e já ter dois ou mais filhos), com uma pre- valência de provável transtorno mental maior do que a esperada e comorbidade de dois ou mais diagnósticos. Dados disponíveis na literatura estimam que $77 \%$ das pacientes com algum diagnóstico psiquiátrico atendido em ambulatórios de ginecologia e obstetrícia dos Estados Unidos não são diagnosticadas pelos seus médicos e, consequentemente, não são tratadas 49 .

A presença da gestante na rede de atenção básica propicia uma oportunidade ímpar, sendo crucial rastrear, diagnosticar e tratar os transtornos mentais nessa etapa da vida, para gerar melhor qualidade de vida para as mulheres, assim como benefícios para seus filhos e a sociedade como um todo. 


\section{Resumo}

Para determinar a prevalência de prováveis transtornos psiquiátricos durante a gravidez e os fatores sociodemográficos associados, utilizou-se o instrumento PRIME-MD, em 712 gestantes (16o a 36o semanas), de 18 unidades básicas de saúde no sul do Brasil. A prevalência de um provável transtorno mental foi de 41,7\%. O transtorno depressivo maior (21,6\%) foi o mais prevalente, seguido pelo Transtorno de Ansiedade Generalizada (19,8\%). Os fatores que mostraram significância com um provável transtorno mental foram: não trabalhar nem estudar RP = 1,25 (IC95\%: 1,04-1,51), não morar com o companheiro $R P=1,24$ (IC95\%: 1,01-1,52), e ter dois ou mais filhos $R P=1,21$ (IC95\%: 1,01-1,46). Houve uma alta prevalência de provável transtorno mental. No período de pré-natal ocorre uma maior frequência de consultas que pode propiciar o rastreamento, o diagnóstico e as abordagens terapêuticas adequadas dos transtornos mentais na rede básica de saúde.

Transtornos Mentais; Gravidez; Atenção Primária à Saúde; Estudos Transversais

\section{Colaboradores}

Todos os autores participaram de todas as etapas na elaboração do artigo.

\section{Agradecimentos}

Ao CNPq (Conselho Nacional de Desenvolvimento Científico e Tecnológico). Aos investigadores do estudo Medida de Consumo Alimentar, Transtornos Mentais e Violência em Gestantes (ECCAGE): Rafael Marques Soares, Andressa Giacomello, Michele Drehmer, Caroline Buss, Juliana Hoffmann, Cristiane Melere e Silvia Ozcariz.

\section{Referências}

1. Department of Mental Health and Substance Dependence, World Health Organization. Prevention and promotion in mental health. Geneva: World Health Organization; 2002.

2. Patel V, Kleinman A. Poverty and common mental disorders in developing countries. Bull World Health Organ 2003; 81:609-15.

3. Cross-national comparisons of the prevalences and correlates of mental disorders. WHO International Consortium in Psychiatric Epidemiology. Bull World Health Organ 2000; 78:413-26.

4. Ansseau M, Dierick M, Buntinkx F, Cnockaert P, De SJ, Van Den Haute M, et al. High prevalence of mental disorders in primary care. J Affect Disord 2004; 78:49-55.

5. Costa AG, Ludermir AB. Transtornos mentais comuns e apoio social: estudo em comunidade rural da Zona da Mata de Pernambuco, Brasil. Cad Saúde Pública 2005; 21:73-9.

6. Linzer M, Spitzer R, Kroenke K, Williams JB, Hahn S, Brody D, et al. Gender, quality of life, and mental disorders in primary care: results from the PRIMEMD 1000 study. Am J Med 1996; 101:526-33.
7. Maragno L, Goldbaum M, Gianini RJ, Novaes HM, Cesar CL. Prevalência de transtornos mentais comuns em populações atendidas pelo Programa Saúde da Família (QUALIS) no Município de São Paulo, Brasil. Cad Saúde Pública 2006; 22:1639-48.

8. Mari JJ. Psychiatric morbidity in three primary medical care clinics in the city of São Paulo. Issues on the mental health of the urban poor. Soc Psychiatry 1987; 22:129-38.

9. Kendell RE, Wainwright S, Hailey A, Shannon B. The influence of childbirth on psychiatric morbidity. Psychol Med 1976; 6:297-302.

10. Gavin NI, Gaynes BN, Lohr KN, Meltzer-Brody S, Gartlehner G, Swinson T. Perinatal depression: a systematic review of prevalence and incidence. Obstet Gynecol 2005; 106 (5 Pt 1):1071-83.

11. Grant KA, McMahon C, Austin MP. Maternal anxiety during the transition to parenthood: a prospective study. J Affect Disord 2008; 108:101-11.

12. van Bussel JC, Spitz B, Demyttenaere K. Women's mental health before, during, and after pregnancy: a population-based controlled cohort study. Birth 2006; 33:297-302. 
13. Evans J, Heron J, Francomb H, Oke S, Golding J. Cohort study of depressed mood during pregnancy and after childbirth. BMJ 2001; 323:257-60.

14. Heron J, O'Connor TG, Evans J, Golding J, Glover $\mathrm{V}$. The course of anxiety and depression through pregnancy and the postpartum in a community sample. J Affect Disord 2004; 80:65-73.

15. Limlomwongse N, Liabsuetrakul T. Cohort study of depressive moods in Thai women during late pregnancy and 6-8 weeks of postpartum using the Edinburgh Postnatal Depression Scale (EPDS). Arch Womens Ment Health 2006; 9:131-8.

16. Rich-Edwards JW, Kleinman K, Abrams A, Harlow BL, McLaughlin TJ, Joffe H, et al. Sociodemographic predictors of antenatal and postpartum depressive symptoms among women in a medical group practice. J Epidemiol Community Health 2006; 60:221-7.

17. Wissart J, Parshad O, Kulkarni S. Prevalence of preand postpartum depression in Jamaican women. BMC Pregnancy Childbirth 2005; 5:15.

18. Lee AM, Lam SK, Sze Mun Lau SM, Chong CS, Chui HW, Fong DY. Prevalence, course, and risk factors for antenatal anxiety and depression. Obstet Gynecol 2007; 110:1102-12.

19 Dayan J, Creveuil C, Herlicoviez M, Herbel C, Baranger E, Savoye C, et al. Role of anxiety and depression in the onset of spontaneous preterm labor. Am J Epidemiol 2002; 155:293-301.

20. Ferri CP, Mitsuhiro SS, Barros MC, Chalem E, Guinsburg R, Patel V, et al. The impact of maternal experience of violence and common mental disorders on neonatal outcomes: a survey of adolescent mothers in Sao Paulo, Brazil. BMC Public Health 2007; 7:209.

21. Orr ST, James SA, Blackmore PC. Maternal prenatal depressive symptoms and spontaneous preterm births among African-American women in Baltimore, Maryland. Am J Epidemiol 2002; 156:797802.

22. Rahman A, Bunn J, Lovel H, Creed F. Association between antenatal depression and low birthweight in a developing country. Acta Psychiatr Scand 2007; 115:481-6.

23. Alder J, Fink N, Bitzer J, Hosli I, Holzgreve W. Depression and anxiety during pregnancy: a risk factor for obstetric, fetal and neonatal outcome? A critical review of the literature. J Matern Fetal Neonatal Med 2007; 20:189-209.

24 Brand SR, Brennan PA. Impact of antenatal and postpartum maternal mental illness: how are the children? Clin Obstet Gynecol 2009; 52:441-55.

25. Faisal-Cury A, Rossi MP. Prevalence of anxiety and depression during pregnancy in a private setting sample. Arch Womens Ment Health 2007; 10:25-32.

26. Faisal-Cury A, Menezes P, Araya R, Zugaib M. Common mental disorders during pregnancy: prevalence and associated factors among low-income women in Sao Paulo, Brazil: depression and anxiety during pregnancy. Arch Womens Ment Health 2009; 12:335-43.

27. Pinheiro SN, Laprega MR, Furtado EF. Morbidade psiquiátrica e uso de álcool em gestantes usuárias do Sistema Único de Saúde. Rev Saúde Pública 2005; 39:593-8.
28. Da-Silva VA, Moraes-Santos AR, Carvalho MS, Martins ML, Teixeira NA. Prenatal and postnatal depression among low income Brazilian women. Braz J Med Biol Res 1998; 31:799-804.

29. Lovisi GM, Lopez JR, Coutinho ES, Patel V. Poverty, violence and depression during pregnancy: a survey of mothers attending a public hospital in Brazil. Psychol Med 2005; 35:1485-92.

30. Pereira PK, Lovisi GM, Pilowsky DL, Lima LA, Legay LF. Depression during pregnancy: prevalence and risk factors among women attending a public health clinic in Rio de Janeiro, Brazil. Cad Saúde Pública 2009; 25:2725-36.

31. Nunes MA, Ferri CP, Manzolli P, Soares RM, Drehmer M, Buss C, et al. Nutrition, mental health and violence: from pregnancy to postpartum Cohort of women attending primary care units in Southern Brazil-ECCAGE study. BMC Psychiatry 2010; 10:66.

32. Spitzer RL, Williams JB, Kroenke K, Linzer M, deGruy III FV, Hahn SR, et al. Utility of a new procedure for diagnosing mental disorders in primary care. The PRIME-MD 1000 study. JAMA 1994; 272:1749-56.

33. Fraguas Jr. R, Henriques Jr. SG, De Lucia MS, Iosifescu DV, Schwartz FH, Menezes PR, et al. The detection of depression in medical setting: a study with PRIME-MD. J Affect Disord 2006; 91:11-7.

34. Work Group to Revise DSM-III, American Psychiatric Association. Diagnostic and statistical manual of mental disorders DSM-III-R. 3rd Ed. Washington DC: American Psychiatric Association; 1987.

35. Barros AJ, Hirakata VN. Alternatives for logistic regression in cross-sectional studies: an empirical comparison of models that directly estimate the prevalence ratio. BMC Med Res Methodol 2003; 3:21.

36 Vigo A. Modelando desfechos comuns: viés e precisão. Cad Saúde Pública 2006; 22:2496-7.

37. Falceto OG, Giugliani ER, Fernandes CL. Influence of parental mental health on early termination of breast-feeding: a case-control study. J Am Board Fam Pract 2004; 17:173-83.

38. Seguin L, Potvin L, St-Denis M, Loiselle J. Chronic stressors, social support, and depression during pregnancy. Obstet Gynecol 1995; 85:583-9.

39. Silva RA, Jansen K, Souza LD, Moraes IG, Tomasi E, Silva GG, et al. Depression during pregnancy in the Brazilian public health care system. Rev Bras Psiquiatr 2010; 32:139-44.

40. Bennett HA, Einarson A, Taddio A, Koren G, Einarson TR. Prevalence of depression during pregnancy: systematic review. Obstet Gynecol 2004; 103:698-709.

41. Ebert MH. Current diagnosis \& treatment psychiatry. 2nd Ed. New York: McGraw-Hill Medical; 2008.

42. Vesga-Lopez O, Blanco C, Keyes K, Olfson M, Grant BF, Hasin DS. Psychiatric disorders in pregnant and postpartum women in the United States. Arch Gen Psychiatry 2008; 65:805-15.

43 Lancaster CA, Gold KJ, Flynn HA, Yoo H, Marcus SM, Davis MM. Risk factors for depressive symptoms during pregnancy: a systematic review. Am J Obstet Gynecol 2010; 202:5-14. 
44. Coutinho ES, de Almeida FN, Mari JJ, Rodrigues LC. Gender and minor psychiatric morbidity: results of a case-control study in a developing country. Int J Psychiatry Med 1999; 29:197-208.

45. Marchesi C, Bertoni S, Maggini C. Major and minor depression in pregnancy. Obstet Gynecol 2009; 113:1292-8.

46. Lima MS, Beria JU, Tomasi E, Conceicao AT, Mari JJ. Stressful life events and minor psychiatric disorders: an estimate of the population attributable fraction in a Brazilian community-based study. Int J Psychiatry Med 1996; 26:211-22.

47. Ludermir AB, Melo Filho DA. Condições de vida e estrutura ocupacional associadas a transtornos mentais comuns. Rev Saúde Pública 2002; 36:213-21.
48. Kroeff LR, Mengue SS, Schmidt MI, Duncan BB, Favaretto AL, Nucci LB. Fatores associados ao fumo em gestantes avaliadas em cidades brasileiras. Rev Saúde Pública 2004; 38:261-7.

49. Spitzer RL, Williams JB, Kroenke K, Hornyak R, McMurray J. Validity and utility of the PRIME-MD patient health questionnaire in assessment of 3000 obstetric-gynecologic patients: the PRIME-MD Patient Health Questionnaire Obstetrics-Gynecology Study. Am J Obstet Gynecol 2000; 183:759-69.

Recebido em 01/Jun/2011

Versão final reapresentada em 23/Ago/2011

Aprovado em 03/Out/2011 